\title{
La politique de mise en valeur des terres arides en Algérie: une lecture en termes d'équité
}

\author{
Ali Daoudi ${ }^{1, *}$, Jean-Philippe Colin ${ }^{1,2}$ et Khadidja Baroud ${ }^{1,3}$ \\ ${ }^{1}$ École Nationale Supérieure Agronomique Kasdi Merbah, El Harrach, Alger, Algérie \\ 2 IRD, UMR GRED, Montpellier, France \\ ${ }^{3}$ Centre de Recherche Scientifique et Technique des Régions Arides, Biskra, Algérie
}

\begin{abstract}
Résumé - Les politiques foncières redistributives suscitent souvent des débats controversés quant à leur équité. La politique de mise en valeur agricole, en cours en Algérie depuis 1983, a donné un nouveau souffle à l'agriculture algérienne et a considérablement élargi les perspectives de développement pour des milliers d'acteurs dans les zones arides du pays. En déverrouillant simultanément l'accès à la terre et à l'eau souterraine publiques, l'État joue un rôle d'allocation, ou dans certains cas de réallocation, des ressources productives. Un réel engouement pour le foncier est observé dans beaucoup de ces zones; ;'accès au foncier public est aujourd'hui l'objet d'enjeux économiques importants. Une étude auprès de différents acteurs impliqués dans cette politique (agriculteurs bénéficiaires et non bénéficiaires de terre, cadres du ministère de l'Agriculture, cadres des structures déconcentrées des administrations publiques concernées, élus locaux), ciblée sur la «petite» mise en valeur, montre comment la question de l'équité de l'allocation des terres publiques dans le cadre de la politique de mise en valeur, est diversement appréciée. Les acteurs locaux, notamment les ayants droit historiques sur les terres objet de mise en valeur, sont les plus sensibles à la question de l'équité, qu'ils apprécient à l'aune de la préservation ou de la perte de leurs droits hérités sur la terre. Les cadres du ministère de l'Agriculture mettent davantage en exergue la question de la réussite de la mise en valeur et considèrent qu'il est plus juste d'attribuer la terre à ceux qui peuvent effectivement la mettre en valeur.
\end{abstract}

Mots clés : Politique foncière / mise en valeur agricole / justice / zones arides / Algérie

\begin{abstract}
Developing arid lands in Algeria: a reading in terms of equity. Redistributive land policies often generate controversial debates about their fairness. The land development policy, ongoing in Algeria since 1983, has given a new push to Algerian agriculture and has considerably widened the development prospects for thousands of actors in the arid zones of the country. By simultaneously unlocking access to public land and groundwater, the State plays a role in allocating, or in some cases reallocating, productive resources. A real land rush is observed in many of these areas, and access to public land is today the subject of important economic stakes. A study of the different types of actors involved in this policy (beneficiaries and non-beneficiaries of land, executives from the Ministry of Agriculture, executives from the deconcentrated structures of the public administrations concerned, local elected representatives), focusing on small-scale land development schemes, shows how, in this context, the fairness of such allocation of public land is perceived. Local actors, particularly those with historical rights to the land being developed, are most sensitive to the issue of equity, which they value in terms of preservation or loss of their inherited land rights. Executives from the Ministry of Agriculture put more emphasis on the success of land development programs and consider it fairer to allocate land to those who can actually develop it.
\end{abstract}

Keywords: Land policy / land development / justice / arid areas / Algeria

\footnotetext{
*Auteur de correspondance : ali.daoudi@edu.ensa.dz
} 


\section{Introduction}

Les réformes agraires redistributives restent d'actualité dans certains pays, principalement dans le cadre de « politiques foncières assistées par le marché» (comme au Brésil, en Afrique du Sud ou au Zimbabwe) (Lahiff et al., 2007), ou dans le cadre de politiques de privatisation totale (transfert de la propriété) ou partielle (transfert du droit d'usage) des terres publiques, comme en Algérie (Daoudi et Colin, 2017).

Cet article porte sur les enjeux de justice inhérents aux politiques de redistribution foncière dans le cadre de la politique de mise en valeur de terres publiques en zones arides, engagées en Algérie à partir de 1983. Dans le contexte algérien, la mise en valeur (MEV) s'entend au sens de mise en culture irriguée de terres jusqu'alors non cultivées, ou seulement exploitées par l'agropastoralisme ou en céréaliculture pluviale extensive. La MEV concerne les terres du domaine privé de l'État, constitué dans les années 1970 par la nationalisation des terres arch (terres steppiques de parcours, sur lesquelles les tribus avaient un droit d'usage traditionnel, ce droit restant reconnu après la nationalisation), de terres communales ou d'espaces non exploités (Sahara). Dans une première phase, la MEV donnait accès à un droit de propriété privée (loi 83-18, dite APFA votée en 1983, portant accession à la propriété foncière agricole par la mise en valeur). Cette cession en toute propriété des terres du domaine privé de l'État a ensuite été limitée, à partir de 1997, et remplacée par un droit d'exploitation formalisé par un contrat de concession de 40 ans. Les superficies cédées, l'octroi ou pas d'aides publiques à la mise en valeur, les catégories de bénéficiaires, ont évolué au gré des différents programmes de MEV.

La lecture en termes de justice proposée dans cet article apporte un nouvel éclairage aux travaux portant sur la MEV en Algérie. Ces travaux relèvent une constance de l'objectif majeur de la politique, l'augmentation de la production agricole et la consolidation de la sécurité alimentaire du pays, et décrivent les changements importants dans son contenu, ses règles, ses formes et ses résultats (Baroud et al., 2018; Bessaoud, 2016; Daoudi et Colin, 2017; Hadeid, 2011; Khiari, 2002 ; Otmane et Kouzmine, 2013). Les changements des droits fonciers sur les terres du domaine privé de l'État ont favorisé l'émergence progressive de différents modèles de $\mathrm{MEV}$, qui cohabitent malgré le caractère parfois contradictoire des principes qui les fondent: concession versus propriété APFA; petite versus grande MEV; MEV aidée ou non par l'État; nouveaux acteurs (investisseurs agricoles urbains) versus anciens acteurs (agriculteurs et agropasteurs). La lecture de ces changements au prisme de la justice s'appuie sur une conception de cette dernière fondée sur la reconnaissance de la pluralité des principes de justice, en raison de la pluralité des normes et valeurs politiques portées par les acteurs concernés (Sen, 2012). Sen note que la coexistence de ces principes concurrents peut donner lieu, en fonction des rapports de pouvoirs locaux, à des compromis négociés, à une subordination d'un principe ou groupe de principes à d'autres, ou à des conflits. Qu'en est-il dans le contexte de la MEV en Algérie?

La section 2 de ce texte présente les politiques de mise en valeur, les grands traits de notre grille d'analyse en termes de justice et la méthodologie adoptée. La diversité des formes des affectations foncières et leur discussion en termes de justice sont présentées respectivement en sections 3 et 4 .

\section{Contexte, cadre conceptuel et méthodologie}

\subsection{La politique de mise en valeur: une dynamique de redistribution foncière d'envergure}

Depuis le lancement de la politique de MEV et jusqu'en 2018, la superficie attribuée totalise (tous programmes confondus) plus de 2,55 millions d'hectares (MADRP, 2018), ce qui en fait une politique de grande envergure. À travers cette politique, les pouvoirs publics facilitent l'accès à trois ressources productives clés: le foncier, l'eau et le financement. Nous insisterons ici sur la composante foncière.

La naissance de la politique de MEV a été actée à travers la loi de l'APFA ( $\left.\mathrm{n}^{\circ} 83-18\right)$ qui ouvre aux nationaux, pour un dinar symbolique, l'accès à la propriété privée de terres du domaine privé de l'État situées pour l'essentiel en zones saharienne et steppique, après une mise en valeur par le bénéficiaire (Baroud et al., 2018). Deux formes ont été définies : à l'initiative des collectivités locales et à l'initiative des candidats à la MEV. Dans ce dernier cas, les candidats doivent détenir un droit d'usage antérieur et non contesté localement sur la terre objet de MEV.

L'État lance ensuite, en 1997, un nouveau programme dit GCA, du nom de l'entreprise publique (Générale des concessions agricoles) en charge des travaux d'aménagement des périmètres à mettre en valeur (décret $n^{\circ} 97-483 \mathrm{du} \mathrm{15/12/}$ 1997). Les parcelles aménagées (de 2 à 5 ha) et totalement équipées (lotissement, forage, plantation fruitière avec parfois un système d'irrigation localisé) sont cédées à des bénéficiaires avec un droit de concession convertible en cession définitive en cas de réussite de l'exploitation.

En 2008, avec la loi $\mathrm{n}^{\mathrm{o}} 08-16$ portant orientation agricole, la concession est décrétée comme unique forme d'attribution foncière pour les terres publiques à vocation agricole aménagées et équipées (pistes agricoles, électrification, certains équipements collectifs comme les forages) par l'État - la concession cesse donc d'être une phase transitoire avant l'accès à la pleine propriété. L'accès à la terre dans le cadre de l'APFA (avec établissement d'un droit de propriété privée) est restreint aux terres «vivifiées » par les particuliers sur leurs fonds propres (Ahmed Ali, 2011). Cette dernière possibilité est ensuite restreinte aux seules régions sahariennes par l'instruction interministérielle $n^{\circ} 402$ de 2011 . En zone de steppe, la concession est, depuis 2008, l'unique mode d'accès au foncier public dans le cadre de la MEV. Deux grands programmes de MEV par la concession, accompagnés de crédits bonifiés, ciblant les zones tant steppiques que sahariennes, ont été lancés en 2011 (Circulaire interministérielle $n^{\circ} 108$ de 2011) et en 2017 (Circulaire interministérielles $\mathrm{n}^{\mathrm{o}} 18-39$ de 2017).

Sur le total des superficies attribuées depuis 1983, moins du tiers serait réellement mis en valeur, au sens de cultivé en irrigué (MADRP, 2018). Le reste est soit en cours de MEV, soit abandonné après échec d'une tentative de MEV ou sans que cette dernière n'ait jamais été engagée. Une opération d'assainissement du foncier agricole attribué dans le cadre 
de la MEV mais non exploité, est en cours depuis 2018 (Circulaire interministérielle $\mathrm{n}^{\circ} 750$ de 2018); aucun bilan n'est publié par le ministère de l'Agriculture à ce jour. Les terres pour lesquelles l'échec de la MEV est définitivement constaté devraient être récupérées et réaffectées par l'État à de nouveaux candidats.

\subsection{La justice comme équité dans l'accès aux ressources}

Les enjeux de justice dans la politique de MEV agricole sont traités ici à travers une combinaison des approches distributive et procédurale de la justice (Jacobs, 1989). La première approche renvoie à l'équité des critères de (re) distribution des ressources et à l'appréciation de l'équité des résultats de cette (re)distribution (dans le contexte qui nous intéresse : critères permettant l'accès à la terre dans le cadre de la politique de $\mathrm{MEV}$, distribution foncière résultante jugée équitable ou non). La seconde repose sur l'équité des processus de prise de décision, des modes opératoires (processus participatif de production et de mise en œuvre de la politique, comme l'impartialité des procédures d'accès à la terre du domaine privé de l'État).

Notre objectif est ainsi de proposer une lecture en termes de justice (i) des règles d'attribution foncière définies par la politique de $\mathrm{MEV}$, et (ii) des conditions effectives d'attribution des terres du domaine privé de l'État. Une attention est portée à la perception des enjeux de justice par les différents acteurs concernés. Nous retiendrons ici la définition de John Rawls (1987 [1971]) de la justice comme équité (fairness) dans la distribution des ressources, mais dans la relecture critique qu'en fait Sen (2012). Grâce à un accord sur les principes qui peuvent gouverner les institutions, Rawls cherche à produire une théorie universaliste de la justice, avec comme objectif la production d'institutions justes régulant l'accès aux «biens premiers »: les libertés fondamentales (de pensée, d'association, de circulation, etc.), les opportunités offertes aux individus, les pouvoirs et prérogatives afférant aux fonctions et positions dans les institutions politiques et économiques, les revenus et la richesse, les bases sociales du respect de soi. Sen, lui, souligne la nécessité incontournable (i) de traiter non seulement des institutions «justes», mais aussi et surtout des comportements réels des acteurs et des «réalisations sociales» concrètes qui vont résulter du jeu de ces institutions; (ii) de reconnaître que certains puissent ne pas se conformer aux normes édictées par ces institutions, et plus largement de reconnaître une interdépendance des rôles des institutions et des comportements d'acteurs dans la réalisation de la justice sociale; (iii) de reconnaître la pluralité des principes de justice, en raison de la pluralité des normes et valeurs politiques portées par les acteurs.

Notre analyse est menée dans un contexte particulier marqué par la coexistence de deux systèmes de droits fonciers en apparence antagoniques. En effet, dans la wilaya de Laghouat, notre site d'étude, les terres susceptibles d'être mises en valeur sont principalement des terres de parcours. Sur ces terres à vocation pastorale, qui relèvent légalement du domaine privé de l'État, les agropasteurs ont des droits d'usage anciens acquis et transmis conformément à un cadre coutumier globalement reconnu par l'État. L'influence de l'existence de ces droits d'usage sur la perception, par les acteurs en présence, des enjeux de justice des redistributions foncières dans le cadre de la MEV demande à être appréciée. Il s'agit pour nous d'identifier les principes selon lesquels les différents acteurs impliqués jugent du caractère juste ou non des critères d'attribution foncière - cette appréciation elle-même pouvant être conditionnée par la perception éventuelle de l'établissement de la domanialité de ces terres, dans les années 1970, comme injuste. Quatre niveaux d'analyse seront distingués : (i) le ministère de l'Agriculture, principal concerné par la conception de la politique de MEV et la production d'outils pour sa mise en œuvre; (ii) la wilaya (préfecture) et les directions déconcentrées des différents départements ministériels concernés, qui disposent d'une marge de manœuvre dans l'interprétation du cadre réglementaire, souvent influencée par leurs propres perceptions; (iii) la commune ou l'assemblée des élus locaux (Assemblée populaire communale, APC), qui jouent un rôle central dans la mise en œuvre de la politique, avec ici encore une certaine marge de manœuvre ; (iv) le périmètre de $\mathrm{MEV}$ et la localité où il est situé, avec les bénéficiaires et les exclus de la politique de MEV.

Nous retenons ainsi une conceptualisation des administrations agraires ou territoriales comme des « champs sociaux semi-autonomes » (Moore, 1973), dont les caractéristiques et le fonctionnement doivent être analysés non seulement en référence aux objectifs et aux moyens qui leur sont formellement assignés, mais aussi au regard de leur capacité à exprimer leurs propres intérêts, leurs valeurs, et à générer leurs propres règles.

\subsection{Méthode}

L'étude a été conduite dans la wilaya de Laghouat, administrativement classée comme wilaya saharienne, mais dont le territoire est partagé entre la steppe (partie centre et nord) et le Sahara (partie sud) (Fig. 1). A Laghouat, la superficie globale attribuée dans le cadre des différents programmes de MEV était, en 2018, de 56654 hectares, dont 54874 dédiés à la petite $\mathrm{MEV}$ (jusqu'à $10 \mathrm{ha}$ ), répartis sur 55 périmètres. La grande $\mathrm{MEV}$ (entre 50 et 500 ha) y est encore très peu développée: en 2018, seuls 20 investisseurs avaient bénéficié d'attributions sur une superficie totale de 1780 hectares, et seulement 10 d'entre eux étaient en cours d'installation au moment de l'enquête. Seuls les périmètres de la petite MEV ont donc été étudiés dans le cadre cette étude. Les appréciations et avis relatifs à la grande $\mathrm{MEV}$ que nous mentionnons dans ce texte expriment l'opinion générale des personnes interrogées, sans référence à un périmètre en particulier.

La collecte de données a reposé sur une combinaison de méthodes et outils :

- Recension des textes légaux relatifs à la politique de MEV et de la littérature sur le sujet.

- Exploitation de données d'archives ou de statistiques.

- Entretiens semi-directifs avec des personnes ressources: responsables (actuels et anciens) des directions centrales du ministère de l'Agriculture chargées du foncier agricole, afin de comprendre le cadre légal et les conditions de conception des programmes de MEV (4) ; agents d'administrations déconcentrées et élus locaux (14): directeurs, chefs 


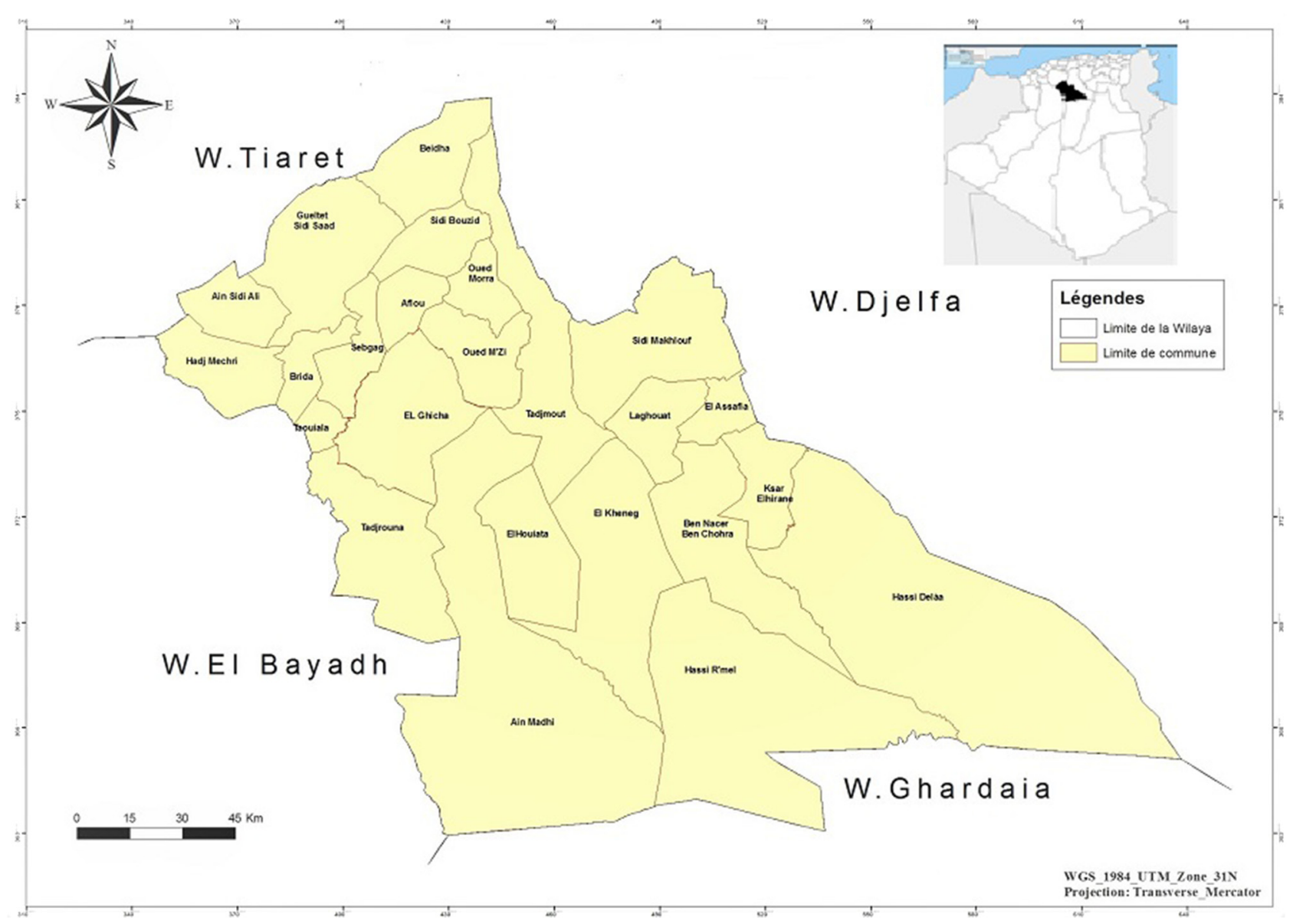

Fig. 1. Carte de la wilaya de Laghouat.

Fig. 1. Map of the wilaya of Laghouat.

services et agents des différentes directions de wilaya (y compris la direction de wilaya de l'Office national des terres agricoles, ONTA), des subdivisions agricoles et communes, afin de clarifier les conditions effectives de mise en œuvre de la politique de MEV par ces acteurs.

- Entretiens semi-directifs avec des bénéficiaires d'attribution foncière dans des périmètres de MEV aménagés par l'État (31), choisis parmi les attributaires et les exclus de 4 périmètres APFA et GCA : Errags (APFA) et Lembed d'Elassafia (GCA), commune de Sidi Makhlouf; Hamda sud (GCA) et Djnein (APFA), commune de Laghouat.

Les entretiens avec les acteurs du ministère de l'Agriculture ont été conduits par Ali Daoudi et Khadidja Baroud; ceux avec les acteurs locaux ont été réalisés par Khadidja Baroud entre janvier et décembre 2018.

\section{Affectations foncières pour la mise en valeur: une diversité de formes et d'enjeux}

Il est important de présenter, pour mieux situer les enjeux de justice, les avantages liés à la redistribution foncière et leur évolution dans le temps. La politique de MEV a eu dès son lancement un caractère redistributif assumé; en plus du foncier, l'État accorde également à travers cette politique des aides financières qui peuvent être conséquentes, équivalant à 10000 euros par hectare mis en valeur dans le cadre du programme lancé en 2011 (Circulaire interministérielle $\mathrm{n}^{\circ} 108$ de 2011). Les avantages auxquels peuvent prétendre les bénéficiaires de la MEV ont beaucoup changé depuis le lancement de cette politique, au début des années 1980. Selon la nature du droit foncier transféré, l'existence ou pas d'un droit d'usage antérieur à l'attribution, l'importance des superficies attribuées et des aides financières à la $\mathrm{MEV}$, le contenu des avantages attribués change et avec lui la perception par les acteurs du caractère juste, ou non, des attributions. Notons que la notion d'équité/justice n'apparaît pas comme un élément constitutif de la composante foncière de la politique de MEV: aucun texte réglementaire n'y fait explicitement référence.

\subsection{Droits fonciers transférés : de la propriété à la concession}

On l'a vu, la MEV peut donner lieu à l'établissement d'un droit de propriété privée (APFA) ou d'un droit d'exploitation (concession). Avant 2008, l'accès au droit de propriété privée était possible pour tous les bénéficiaires de terres publiques ayant mené à bien leur projet de $\mathrm{MEV}$, y compris les 
bénéficiaires de concessions dites GCA. L'accès à ce droit est depuis devenu plus restrictif: seule la MEV sur fonds propres et dans les zones sahariennes le permet encore. Ce changement important de règle du jeu est différemment apprécié par les acteurs.

Les candidats à la mise en valeur, notamment les habitants des régions steppiques, y voient une forme d'injustice à l'égard des nouveaux bénéficiaires, par rapport aux anciens : le même effort de vivification de la terre ne donne pas les mêmes droits. Un concessionnaire, exclu de l'APFA, demande ainsi au fonctionnaire de l'Office national des terres agricoles (ONTA): "Pourquoi vous m'intégrez dans la concession, alors qu'à d'autres vous donnez un droit de propriété privée? Pourquoi, et sur la base de quels critères, vous faites ce choix? Moi, en concession, peut-être que j'investis plus dans mon exploitation qu'un bénéficiaire de l'APFA» (propos rapportés par un agent de l'ONTA).

Les candidats à la $\mathrm{MEV}$ dans les régions sahariennes, notamment les petits, voient quant à eux une forme d'exclusion dans la limitation de l'accès au droit de propriété aux seules personnes capables de financer le projet de MEV par leurs propres moyens.

Pour les décideurs politiques, ce changement permet de concilier le principe d'équité intergénérationnelle avec le souci d'efficience productive. Les réserves foncières à potentiel agricole se réduisent, y compris sur les vastes étendues de la steppe et du Sahara. Poursuivre le processus de privatisation de ces terres est un choix dorénavant rejeté par les décideurs politiques - le Président de la République au premier chef, qui a personnellement tranché, en 2008, pour le modèle de la concession. Les arguments justifiant l'arrêt de la privatisation des terres du domaine privé de l'État obéissent à une vision politique de la justice selon laquelle les terres publiques sont un patrimoine national commun, qu'il serait injuste, à l'égard des générations futures, de privatiser.

"Au lancement de la politique de mise en valeur, l'État voulait rendre attractive cette entreprise économique difficile; la cession de la propriété foncière était un argument dans ce sens. L'accroissement considérable de la demande sur le foncier, enregistré durant les années 2000, a motivé l'adoption du régime de concession pour freiner le processus de privatisation des terres publiques sans ralentir la dynamique de mise en valeur. Des cercles politiques puissants s'opposent à la privatisation des terres agricoles publiques» (ancien Directeur général de l'ONTA).

Pour les cadres du ministère de l'Agriculture, le choix du droit de concession de 40 ans permet, sans aliéner la terre, de sécuriser les droits des investisseurs et donc de les inciter à s'engager pleinement dans leur projet agricole. Dans les termes d'un responsable de la Direction de l'organisation foncière et de la protection du patrimoine du ministère (DOFPP), le choix de la formule de la concession, notamment pour les investisseurs, est un changement positif: "On était dans une vision d'attribution, on est dans une vision de valorisation; on était dans une vision d'occupation du sol, on est dans une vision d'optimisation économique du sol».

Le maintien de l'APFA au Sahara serait justifié, selon ces mêmes responsables, à la fois par l'importance des investissements à engager pour la mise en valeur, et surtout par une appréciation des enjeux fonciers qui seraient très faibles dans cette partie du pays. Dans la pratique, tous les programmes de
MEV engagés et soutenus par l'État depuis 2011 sont entrepris sous le régime de la concession. Le maintien de l'APFA semble être un choix provisoire, le temps de régulariser les anciens programmes; l'absence de nouvelles attributions consolide cette interprétation.

\subsection{Affectations foncières : entre transfert redistributif et consolidation/formalisation de droits antérieurs}

Les terres du domaine privé de l'État attribuées pour être mises en valeur peuvent être déjà anthropisées. Les terres à vocation pastorale, y compris les parcours présahariens, sont utilisées de longue date par les agropasteurs, qui ont de ce fait un droit d'usage sur ces terres, reconnu par la loi (Code pastoral de 1975). Dans ce cas, l'attribution des terres pastorales ne correspond pas toujours à un simple transfert du droit de propriété de l'État vers les candidats qui ont réussi la MEV, il peut s'agir également d'une redistribution, les anciens usagers perdant leur droit d'usage au bénéfice de nouveaux attributaires. Le transfert de droits fonciers dans le cadre de la MEV peut ainsi donner lieu à trois cas de figure :

- un simple transfert du droit (d'exploitation ou de propriété) de l'État vers les bénéficiaires de la MEV (via la concession ou un droit de propriété privée), lorsqu'aucun droit d'usage antérieur n'est détenu par des exploitants cas exclusivement rencontrés dans les zones sahariennes jamais exploitées ;

- un transfert redistributif (ré-allocatif), lorsque la terre en question était antérieurement utilisée de manière régulière ou occasionnelle comme pâturage par d'autres exploitants que les bénéficiaires du transfert; les premiers perdent alors leur droit d'usage antérieur;

- une individualisation et une formalisation (voire une privatisation dans le cas de l'APFA), dans le cas où les bénéficiaires du transfert de propriété détenaient, individuellement ou collectivement, un droit d'usage antérieur sur la terre concernée.

Les anciens détenteurs de droits d'usage (même pour des usages irréguliers) perçoivent l'allocation foncière dans le cadre de la MEV comme injuste, quand elle ne leur bénéficie pas. Pour éviter l'exacerbation de ce sentiment, deux mesures de la procédure d'attribution foncière ont été retenues: (i) le choix des périmètres à mettre en valeur est usuellement laissé aux maires et élus locaux, qui ciblent, souvent en impliquant une partie de la population, les territoires les moins utilisés et sur lesquelles les droits historiques des habitants sont les moins forts (comme les anciennes terres communales de l'époque coloniale, versées dans le domaine privé de l'État en 1971), et (ii) l'institutionnalisation de la priorité des riverains des périmètres de la MEV dans les attributions ; plus largement, les habitants de la commune concernée sont également prioritaires sur les candidats allogènes.

Ces deux mesures sont appliquées pour les périmètres de la petite MEV; elles ont permis l'identification et la distribution, sans conflits majeurs, de centaines de milliers d'hectares à travers les wilayas steppiques. L'implication des acteurs locaux a donc joué rôle important dans la légitimation du processus d'allocation des terres publiques destinées à la MEV. Paradoxalement, ces acteurs locaux perdent complètement la 
main sur le processus d'attribution pour la grande MEV - des attributions dans lesquelles la priorité est accordée aux investisseurs, souvent d'origine urbaine. Lorsque la part de la grande MEV était très limitée, et lorsque les terres qui étaient attribuées étaient situées dans des zones isolées et peu accessibles, le contrôle par l'administration régionale (wilaya) et centrale n'était pas vraiment contesté. Aujourd'hui, les habitants de certaines communes où les projets de nouveaux périmètres de MEV sont identifiés s'érigent contre ces derniers et mettent les élus locaux, censés représenter leur intérêt, devant leurs responsabilités. Le sentiment d'injustice que suscite la grande MEV dans ces communes n'est pas nécessairement dû à l'origine des bénéficiaires (grands investisseurs urbains), mais vient plutôt du sentiment que ces derniers s'accaparent des ressources locales sans contreparties pour le territoire. À Laghouat, la résistance des acteurs locaux (agropasteurs et élus) à la grande MEV est reconnue par les cadres des structures agricoles que nous avons interrogés.

\subsection{Superficies transférées: une dynamique de polarisation}

Selon les superficies attribuées, trois types de MEV sont généralement distingués : la petite $\mathrm{MEV}$ (jusqu'à 10 ha), la grande $\mathrm{MEV}$ (de 50 à 500 ha) et la très grande MEV (plusieurs milliers d'ha, que l'on qualifiera de méga-MEV), introduite depuis 2011 dans le cadre de la concession. La coexistence d'une diversité de modèles de $\mathrm{MEV}$ semble une réponse pragmatique à une demande différenciée; chacun des modèles est adapté à une catégorie de demandeurs, selon ses capacités d'investissement. Une tendance à la polarisation de la politique d'attribution est toutefois à souligner, avec l'augmentation, à l'échelle nationale, des superficies réservées à la très grande $\mathrm{MEV}$ - la petite MEV restant majoritaire en termes de nombre de bénéficiaires.

Cette inégalité des attributions questionne le caractère équitable de la politique, avec un réel enjeu en termes de justice distributive, car la multiplication des très grandes attributions risque, à terme, d'épuiser les réserves foncières et de limiter les opportunités d'accès au foncier pour les milliers de jeunes arrivant en âge de développer une activité productive autonome. Cette appréciation est largement partagée par les agriculteurs interrogés et les autres acteurs locaux.

L'existence simultanée de plusieurs modalités d'attribution foncière est présentée par les cadres centraux du ministère de l'Agriculture comme une forme d'adaptation aux réactions et exigences, souvent contradictoires, des acteurs concernés par la MEV. Ainsi, au niveau des communes steppiques et sahariennes disposant de réserves foncières potentiellement exploitables par l'agriculture, la multiplication des périmètres de petite $\mathrm{MEV}$ est une réponse à une demande locale de plus en plus grande.

La restriction de la grande MEV au seul Sahara résulte en partie de la pression des agropasteurs de la steppe, qui voient dans la grande MEV une menace pour la pérennité de leur activité d'élevage, et surtout pour leurs droits traditionnels sur les parcours steppiques. Quant à l'extension des superficies réservées à la grande $\mathrm{MEV}$ et à l'introduction de la méga-MEV au Sahara, elles sont, en grande partie, une réponse à la demande émanant des grands investisseurs nationaux.

\subsection{De la mise en valeur sur fonds propre à la mise en valeur aidée}

Dans l'esprit de la loi de l'APFA, actant la naissance de la politique de MEV, l'accès au droit de propriété est conditionné par la vivification de la terre par l'effort personnel. Très vite, des programmes d'aide ont cependant été mis en place pour accompagner les candidats à la MEV ne disposant pas de moyens suffisants. Ces aides ont certes facilité la MEV pour beaucoup de candidats, mais ont également attiré des capteurs de rente. L'accès à ces aides n'a pas été le même pour tous ; trois cas de figure se distinguent:

- la MEV sans aide de l'État : c'est généralement le cas de la MEV engagée à l'initiative des exploitants sur des terres sur lesquelles ils ont un droit d'usage non contesté localement ;

- la MEV avec des aides directes à concours définitif (aménagement du périmètre, forage, système d'irrigation, plantation): l'importance de ces aides varie d'un programme à un autre;

- la MEV avec crédits bonifiés dédiés : c'est en particulier le cas des projets (indépendamment de leur importance) lancés dans le cadre de la circulaire interministérielle $\mathrm{n}^{\mathrm{o}} 108$ de 2011.

Les avantages financiers accordés par l'État aux candidats à la $\mathrm{MEV}$ et les écarts dans le traitement des demandes, ainsi que les pratiques de passe-droits, mettent à mal la perception de cette politique par les exclus de ces aides.

Un exploitant installé sur une terre achetée, après avoir été exclu d'une attribution publique, exprime son point de vue sur l'accès aux aides publiques: "Notre administration est très bureaucratisée... Ses agents créent des obstacles aux candidats aux aides afin de réserver ces avantages à des privilégiés... La plupart des agriculteurs ordinaires appréhendent n'importe quelle démarche administrative (demande de sortie de la commission [pour constater la mise en valeur de la terre et obtenir la levée de la condition résolutoire], demande de subvention, demande d'autorisation de forage, etc.). Pour les riches et ceux qui détiennent le pouvoir, toutes ces procédures sont facilitées par leurs amis au sein de l'administration ».

Ce constat a été également établi par Otmane et Kouzmine (2013) dans la région du Touat-Gourara-Tidikelt, dans la wilaya d'Adrar: "les premiers pionniers sont originaires de la région (...); des élus communaux ou des personnes aisées financièrement proches de l'administration sont les premiers à avoir tenté l'expérience pour tirer profit d'un projet fortement financé par l'Etat».

\section{Critères d'accès au foncier: une évolution cumulative}

L'équité d'une politique se lit au travers de ses objectifs, son public cible, ses procédures et leur mise en œuvre effective. Elle se lit également à travers la perception des acteurs qui la subissent ou en bénéficient. Dans cette section, le focus est mis sur les critères de sélection des bénéficiaires et leur perception par les acteurs en jeu. Comme pour tous les 
paramètres étudiés jusqu'à présent, ces deux composantes ont connu un changement dans le temps, en écho au changement des conditions et priorités économiques, sociales et politiques.

\subsection{Conditions de base pour l'accès au foncier pour la mise en valeur}

Pour accéder au foncier dans le cadre de la MEV, il faut être citoyen algérien et porteur d'un projet agricole réalisable. Ces deux conditions n'ont pas changé depuis le lancement de la $\mathrm{MEV}$ et en font, a priori, une politique inclusive de toutes les catégories socioprofessionnelles, du moment qu'aucun seuil de superficie ni d'investissement n'est défini comme critère d'éligibilité. Une troisième condition annoncée dans la loi de l'APFA, la possession des moyens de la MEV, semble par contre discriminer les acteurs ayant un projet, mais sans les moyens pour le mettre en œuvre. Ce critère est-il pour autant perçu par les acteurs comme inéquitable dans une société où traditionnellement l'accès à la propriété de la terre se mérite par l'effort de la vivification?

Ce critère n'a en fait pas été respecté, l'État s'étant engagé, dès le lancement de la politique, dans le financement, au moins partiel, de certains projets de MEV. Dans un premier temps, ces aides étaient orientées vers les bénéficiaires de la petite $\mathrm{MEV}$, puis ont été élargies progressivement aux autres catégories. Ce financement public des investissements inhérents à la MEV semble constituer un écart par rapport à la règle de vivification-un écart d'autant plus inéquitable que ces subventions sont proportionnelles aux superficies attribuées. Dans sa volonté d'inclure tous les acteurs, l'État peut ainsi créer des perceptions d'iniquité, ici au regard de l'accès aux aides publiques.

Sans contester le principe de la vivification comme condition d'accès à une terre publique, les agriculteurs interrogés mettent en avant le rôle clé que l'État doit jouer dans l'aide à la MEV. Cette perception est probablement influencée par le discours dominant considérant la $\mathrm{MEV}$ comme une entreprise d'intérêt général, de par son importance dans la consolidation de la sécurité alimentaire du pays, et par l'implication effective de l'État dans le soutien de l'agriculture d'une manière générale - pourquoi alors exclure la MEV ?

Les cadres du ministère de l'Agriculture interviewés justifient le financement public de la MEV par le souci de l'État de promouvoir cette dernière et de la rendre accessible à tous. Un financement public qui ne doit être, selon ces cadres, qu'un appoint aux moyens des candidats à la $\mathrm{MEV}$, petits et grands. La limitation de l'accès à la propriété foncière aux seuls candidats ayant financé leur projet de MEV sur fonds propres semble correspondre à un compromis pragmatique.

\subsection{Les figures du bénéficiaire légitime selon les acteurs: à chacun sa légitimité}

Dès le lancement de la politique de MEV, l'État a interpellé les communes disposant de réserves foncières adéquates, pour délimiter et aménager (lotissement, ouverture de pistes, électrification et forages collectif, le cas échéant, etc.) des périmètres à attribuer aux candidats à la MEV. Il s'est alors posé la question de comment départager ces candidats. Les habitants des zones d'implantation des périmètres de MEV ont constitué la première des catégories à être priorisée, et ce jusqu'à aujourd'hui; parmi ces habitants, les anciens usagers de la terre objet de la mise en valeur sont encore plus avantagés. D'autres catégories socioprofessionnelles ont été privilégiées par les différents gouvernements qui se sont succédé depuis la fin des années 1980, en fonction des conjonctures politique et économique et de leur orientation idéologique. À la fin des années 1980, dans un contexte d'instabilité sociopolitique sur fond de crise économique aiguë, le gouvernent a mis en place un programme de MEV dédié exclusivement aux chômeurs, globalement jeunes. Cette catégorie a depuis bénéficié d'un traitement particulier dans tous les programmes de MEV (Daoudi et Colin, 2019). D'autres catégories socioprofessionnelles ont été ou sont ciblées, avec parfois des programmes qui leur sont spécifiquement dédiés : les diplômés de la formation agronomique (2000) et les investisseurs porteurs de grands projets agricoles céréaliers (programme lancé en 1992) ou de mégaprojets agroindustriels intégrés (2014 et 2020). Les anciens moudjahidines (combattants de la guerre de libération nationale) ont fait partie des catégories privilégiées, notamment dans les programmes mis en œuvre durant les années 1980 et 1990.

Dans la pratique, des écarts importants sont constatés, et les listes des bénéficiaires des périmètres de MEV contiennent souvent des acteurs relevant de catégories non éligibles, à travers des prête-noms le cas échéant. Des fonctionnaires et des membres des professions libérales sont parmi ces «indus bénéficiaires $\gg$.

Les textes ne font pas référence au genre des candidats, et ne promeuvent pas spécifiquement les attributions aux femmes - le nombre de femmes bénéficiaires est de fait quasi nul et, lorsqu'elles apparaissent, ce sont des prête-noms, selon nos enquêtes.

La question du choix des bénéficiaires des attributions foncières a été, et reste, particulièrement sensible dans les régions steppiques, où la terre avait déjà des usagers. L'affectation dans le cadre de la MEV correspond dans ce cas à une redéfinition et/ou une réallocation de ces anciens droits, on l'a vu. Pour qu'elle soit acceptée, la redistribution foncière doit être considérée comme juste par les acteurs en jeu, tout particulièrement les acteurs qui utilisaient ces terres. Dans la pratique, le choix des bénéficiaires est le résultat de compromis dans lesquels chaque catégorie d'acteurs tente de faire valoir son point de vue. Les listes des bénéficiaires des périmètres aménagés par l'État sont souvent le reflet du rapport de force prévalant localement au moment de l'attribution. Lorsque le périmètre est situé sur les terres de parcours habituellement utilisées par une famille élargie ou sur les terres d'une fraction de tribu organisée et puissante, l'attribution se fait entre ses membres. Quel que soit le critère de sélection mis en avant par l'administration, la famille/fraction s'adapte. Si la priorité est accordée aux jeunes chômeurs dans le programme mis en œuvre par l'administration, la famille les trouvera parmi ses membres, souvent avec une interprétation large des critères. Pour d'autres périmètres, les listes sont plus hétérogènes, on peut y trouver des bénéficiaires non éligibles (fonctionnaires) ou originaires d'autres communes; lorsque c'est le cas, les acteurs locaux y voient la main de l'administration (maire, sous-préfet, wali, directions techniques). 
Deux principes fondent la perception de la justice dans les discours des acteurs de la MEV (acteurs institutionnels et agriculteurs bénéficiaires et non bénéficiaires): le droit antérieur sur la terre et l'effort de mise en valeur. Est vu comme légitime tout candidat réunissant ces deux conditions, à défaut l'une des deux. Pour le cas des candidats ne réunissant que l'une des deux conditions, les avis des acteurs divergent. Les acteurs institutionnels (cadres des structures publiques) considèrent que le mérite revient au candidat ayant un projet de $\mathrm{MEV}$ viable et les moyens de le mener à bien. Selon cette perspective, l'investisseur agricole est l'archétype du candidat légitime. Le principe de légitimation est ici la contribution effective à la valorisation de la terre publique et au développement économique de la région et du pays. La majorité des cadres des institutions publiques interrogés adhèrent à ce principe et critiquent les attributions dites «sociales». Un chef de service à la direction des domaines fonciers de Laghouat exprime son point de vue sur le sujet: "Personnellement, je suis contre les critères d'attribution retenus par la commission technique, je préfère accorder la terre à une personne capable financièrement et apte à travailler la terre, et non à une personne qui va l'accaparer 40 ans sans rien faire ». Les mêmes arguments sont avancés par un ancien chef de service de la mise en valeur à la Direction des services agricoles de Laghouat: "Sincèrement, celui qui veut faire de la mise en valeur, il doit avoir de l'argent. Pour moi, ce n'est pas de la justice d'attribuer la terre à une personne qui n'a pas d'argent pour le faire. Malheureusement le programme de MEV a été plus mobilisé pour consolider la paix sociale». Un autre cadre du secteur agricole, le subdivisionnaire des services agricoles de la commune d'Aïn Madhi abonde dans le même sens : "Je considère que la terre doit être attribuée à des gens capables financièrement. Attribuer un périmètre aménagé par l'État, à coups de milliards, à 50 chômeurs qui n'ont pas même de quoi payer les frais de leurs déplacements vers leur parcelle, ce n'est pas rationnel ».

Pour les habitants des zones concernées par la MEV, la priorité revient à l'ayant droit sur les terres ciblées par les périmètres. Cette perception est fondée sur une réalité souvent éludée par les concepteurs des politiques, par méconnaissance ou légalisme excessif : la terre en steppe n'est pas vacante, tous les parcours ont leurs usagers, avec des droits connus et reconnus. Les détenteurs de ces droits sont considérés localement comme étant les bénéficiaires légitimes de tout projet de MEV de ces terres.

Un bénéficiaire d'une attribution foncière dans le cadre de la circulaire $\mathrm{n}^{\mathrm{o}} 108$ explique comment le choix des bénéficiaires d'un périmètre réalisé sur des terres exploitées par sa famille a été fait: "Après avoir aménagé le périmètre qui se trouvait sur nos terres, la commune nous a demandé de désigner 22 candidats. On s'est organisés entre les membres de notre famille élargie et nos demandes ont été acceptées... Aucun étranger n'a été accepté sur nos terres, c'est impossible... Personne en dehors des membres de la famille n'a eu l'audace de candidater ». Un bénéficiaire d'un autre périmètre (GCA) explique comment sa famille résiste à l'attribution de lots de terre, sans son consentement, à des anciens moudjahidines étrangers à la famille, le périmètre étant situé sur les terres exploitées par la famille depuis des générations: "Nous n'avons pas accepté l'attribution de parcelles de nos terres aux moudjahidines étrangers à la famille. Nous exploitons ces terres depuis toujours, ce sont les terres de nos ancêtres et sur lesquelles nous sommes installés... Maintenant, d'autres viennent les prendre, est-ce que nous acceptons? Bien sûr que non, c'est de la Hogra (de l'injustice) ». À ce jour, ces parcelles font l'objet de litiges et ne sont pas exploitées par leurs attributaires, certains ont officiellement abandonné, d'autres s'accrochent.

La priorité des ayants droit coutumiers dans les attributions foncières est reconnue par les acteurs institutionnels en charge de la MEV, même si beaucoup mettent en cause leurs capacités à réussir la MEV. Le délégué agricole à la commune de Laghouat considère ainsi : "Logiquement et équitablement, les premiers bénéficiaires des terres de la mise en valeur doivent être les populations locales des communes, les résidents des périmètres notamment. Celui qui est déjà installé sur un territoire, tu ne peux pas l'expulser, il faut le prendre en considération ». Ce même délégué enchaîne : "mais en réalité, pour cette catégorie de bénéficiaires (les ayants droithistoriques), généralement ils laissent leur parcelle en friche ou les vendent. À titre d'exemple, dans le périmètre de Hamda, totalement affecté aux ayants droit, $80 \%$ des parcelles ont été vendues ».

Un ancien directeur de projet à la GCA note, au sujet des attributions dans le cadre d'un périmètre de concession qu'il a géré: "Il faut être réaliste, un natif, même s'il n'a pas les moyens de mettre en valeur la terre, le fait qu'il soit résident lui donne la priorité. Imagine que l'Etat installe à sa place d'autres gens, qui ont les moyens, où va partir le natif? S'il ne bénéficie pas d'attribution sur ses terres coutumières, où il va bénéficier?».

La robustesse de cet argument s'affaiblit pour les terres sur lesquelles les droits ne sont pas renforcés par des usages fréquents (parcours éloignés des zones d'habitation et plus encore pour les parcours présahariens) et/ou qui restent détenus par des tribus ou fractions de tribu très larges. Dans ces cas, tous les membres de la tribu et/ou de la fraction sont des ayants droit; ce critère ne permet pas de les départager. Souvent, d'autres critères interviennent et leur prévalence dépend des rapports de force au niveau local.

Pour les acteurs locaux, agriculteurs et élus, le jeune chômeur est la figure consensuelle du bénéficiaire légitime; aucun acteur ne remet en cause le principe d'un droit d'accès des jeunes chômeurs aux attributions foncières. D'abord, pour des raisons objectives : ces jeunes ont besoin de travail et leur accès aux programmes de MEV est donc légitime. Ensuite, parce qu'en zone rurale, chaque famille peut avoir son jeune chômeur et peut donc bénéficier de ces programmes. Par contre leur capacité effective à mettre en œuvre des projets de $\mathrm{MEV}$ est souvent contestée par les cadres de l'administration agricole : "Le critère d'attribution pour des chômeurs, dans la GCA et autres programmes, est discutable. Telles que ces attributions sont conçues, elles sont contradictoires; tu ne peux pas demander à un chômeur de travailler une terre en friche alors qu'il n'a rien pour le faire. Même si tu lui donnes une exploitation clé en main, il n'a pas de quoi la faire fonctionner» (Directeur de projets GCA). 


\section{Conclusion}

Cette étude avait comme objectif de questionner les critères d'attribution foncière, de décrypter leur perception par les acteurs et d'apprécier la «politique en actes». L'histoire récente de la constitution du domaine privé de l'État, comme symbole constitutif du recouvrement de l'indépendance nationale, a contribué à la formation d'une perception largement partagée au sein de la société algérienne, notamment dans le milieu rural, considérant la terre publique comme un bien commun. Le foncier n'est donc pas perçu comme un simple bien privé de l'État que ce dernier peut valoriser librement. Dans l'esprit de beaucoup, les terres publiques leur appartiennent et leur éventuelle privatisation doit profiter à tous, même si c'est au détriment de l'efficience productive. Dans ce contexte, la lecture des politiques foncières sous le prisme de la justice revêt tout son intérêt.

Au regard des procédures, la redistribution foncière dans le cadre de la petite MEV paraît globalement inclusive et équitable. Les deux textes de lois (loi de l'APFA de 1983 et loi d'orientation agricole de 2008) qui offrent les bases légales de la redistribution des droits sur le foncier public, définissent à grands traits le profil du bénéficiaire des attributions de terres publiques : un citoyen algérien porteur d'un projet de mise en valeur. Aucune distinction n'est donc légalement instituée, ce qui permet au pouvoir exécutif d'orienter les attributions en fonction des objectifs économiques et politiques du moment, expression des rapports de force sur la scène politique et économique. Différents programmes de MEV ont été ainsi mis en place par les différents gouvernements qui se sont succédé depuis les années 1980, chacun avec un ensemble de textes règlementaires spécifiques ciblant les catégories d'acteurs jugées alors prioritaires. Une constante est relevée: l'intérêt accordé à la légitimation locale des programmes de redistribution foncière. Les critères d'attribution, comme la procédure de mise en œuvre de l'ensemble de l'opération de redistribution, sont définis pour garantir globalement cette légitimation locale. En matière d'attribution, la priorité est accordée aux agropasteurs, aux petits agriculteurs et aux jeunes chômeurs habitants dans les territoires concernés par la MEV, et à une époque aux anciens moudjahidines. Ces bénéficiaires tirent leur légitimité d'un droit d'usage antérieur sur la terre objet de MEV (les agropasteurs), de leur besoin objectif en terre (petits agriculteurs locaux sous contrainte foncière), de l'accès à des ressources créatrices de revenus (jeunes sans emploi), ou de leur légitimité politique historique (anciens moudjahidines).

La mise en œuvre de l'opération d'attribution foncière dans le cadre de la MEV est du ressort de l'Assemblée populaire communale élue (APC), censée incarner la légitimité populaire et défendre l'intérêt général des habitants de la commune. Une assemblée qui toutefois reflète les rapports de force au niveau local et peut donc jouir d'une légitimité toute relative, notamment en défendant des intérêts catégoriels. Le pouvoir des élus locaux sur le processus d'attribution foncière est toutefois fortement limité par l'administration qui impose ses décisions, souvent sous des prétextes techniques peu maîtrisés par les élus.
En définitive, les politiques de MEV semblent équitables en termes d'approche distributive de la justice quant aux critères d'allocation de la ressource foncière; en termes de conséquences sur la distribution foncière, la rupture majeure intervient à partir de 2011 (la circulaire 108), qui privilégie dans des proportions considérables la méga-MEV. La présence «d'indus-bénéficiaires » (fonctionnaires, commerçants, etc. ayant bénéficié de dotations foncières sur les périmètres aménagés par l'État) ne semble a priori pas avoir eu d'effets d'exclusion sur d'autres catégories d'acteurs, mais ce point reste à vérifier. Relativement aux dimensions procédurales de la justice, l'équité des modes opératoires n'est questionnable que pour la grande et surtout la très grande $\mathrm{MEV}$, qui échappent totalement au contrôle des acteurs locaux.

Remerciements. Cette étude s'inscrit dans le cadre du projet ANR-14-CE18-0001 JASMINN (Programme AAP Générique 2014): Protection du foncier agricole périurbain en Méditerranée: enjeux de justice et innovations foncières.

\section{Références}

Ahmed Ali A. 2011. La législation foncière agricole en Algérie et les formes d'accès à la terre. Options Méditerranéennes 66 : $35-51$.

Baroud K, Colin JP, Daoudi A. 2018. La politique d'accès à la propriété privée des terres mises en valeur en zones arides en Algérie. Éléments de discussion. Economie rurale 363: 81-98. DOI: 10.4000 /economierurale. 5423 .

Bessaoud O. 2016. Les réformes agraires post-coloniales au Maghreb : un processus inachevé. Revue d'histoire moderne et contemporaine 63(4/4 bis): 115-136. DOI: $10.3917 /$ rhmc.634.0115.

Daoudi A, Colin JP. 2017. Construction et transfert de la propriété foncière dans la nouvelle agriculture steppique et saharienne en Algérie. In: Guignard D, ed. Propriété et Société en Algérie contemporaine. Aix-en-Provence: Iremam, OpenEdition Books, pp. 158-176.

Daoudi A, Colin JP. 2019. Installation des jeunes dans le cadre des politiques de mise en valeur en Algérie: portées et limites. In : Merlet M, Lévesque R, Rangé C, Benkahla A. (dir.). Structures agraires et accès des jeunes à la terre: gestion intrafamiliale du foncier et stratégies d'autonomisation des jeunes. Regards sur le foncier no. 7, Comité technique «Foncier \& développement». Paris : AFD, MEAE, pp. 99-111.

Hadeid M. 2011. La politique de mise en valeur agricole en milieu steppique algérien : un essai de bilan dans les Hautes Plaines sud oranaises (Algérie). Insaniyat 51-52: 99-118. DOI: 10.4000/ insaniyat. 12723 .

Jacobs H. 1989. Social Equity in Agricultural Land Protection. Landscape and Urban Planning 17: 21-33. DOI: 10.1016/ 0169-2046(89)90064-9.

Khiari A. 2002. Une région pionnière dans le Sahara algérien: El Ghrouss. Méditerranée 3-4: 27-30. DOI: 10.3406/ medit.2002.3256.

Lahiff E, Borras S, Kay C. 2007. Market-Led Agrarian Reform: policies, performance and prospects. Third World Quaterly 28(8): 1417-1436. DOI: 10.1080/01436590701637318. 
MADRP. 2018. Le foncier agricole par les chiffres. Alger: Ministère de l'Agriculture, du Développement rural et de la pêche.

Moore SF. 1973, Law and social change: the semi-autonomous social field as an appropriate subject of study. Law and Society Review 7: 719-746. DOI: 10.2307/3052967.
Otmane T, Kouzmine Y. 2013. Bilan spatialisé de la mise en valeur agricole au Sahara algérien. Cybergeo: European Journal of Geography. Espace, Société, Territoire 632. DOI: 10.4000/ cybergeo.25732.

Rawls J. 1987. Théorie de la Justice, (1 ${ }^{\mathrm{e}}$ éd. 1971). Paris : Le Seuil. Sen A. 2012. L'idée de justice. Paris : Flammarion.

Citation de l'article : Daoudi A, Colin J-P, Baroud K. 2021. La politique de mise en valeur des terres arides en Algérie : une lecture en termes d'équité. Cah. Agric. 30: 4. 